Research Article

\title{
Generalization of the Convective Flow of Brinkman-Type Fluid Using Fourier's and Fick's Laws: Exact Solutions and Entropy Generation
}

\author{
Nadeem Ahmad Sheikh, ${ }^{1}$ Dennis Ling Chuan Ching, ${ }^{1}$ Ilyas Khan $\mathbb{D}^{2}{ }^{2}$ \\ and Hamzah bin Sakidin ${ }^{1}$ \\ ${ }^{1}$ Fundamental and Applied Science Department, Universiti Teknologi PETRONAS, Seri Iskandar, Perak 32610, Malaysia \\ ${ }^{2}$ Faculty of Mathematics and Statistics, Ton Duc Thang University, Ho Chi Minh City 72915, Vietnam \\ Correspondence should be addressed to Ilyas Khan; ilyaskhan@tdtu.edu.vn
}

Received 13 September 2020; Revised 3 October 2020; Accepted 15 December 2020; Published 30 December 2020

Academic Editor: Sang-Bing Tsai

Copyright $\odot 2020$ Nadeem Ahmad Sheikh et al. This is an open access article distributed under the Creative Commons Attribution License, which permits unrestricted use, distribution, and reproduction in any medium, provided the original work is properly cited.

\begin{abstract}
A new scheme to formulating the Caputo time-fractional model for the flow of Brinkman-type fluid between the plates was introduced by using the generalized laws of Fourier and Fick. Within a channel, free convection flow of the electrically conducted Brinkman-type fluid was considered. A newly generated transformation was applied to the heat and mass concentration equations. The governing equations were solved by the techniques of Fourier sine and the Laplace transforms. In terms of the special function, namely, the Mittag-Leffler function, final solutions were obtained. The entropy generation and Bejan number are also calculated for the given flow. To explain the conceptual arguments of the embedded parameters, separate plots are represented in figures and are often quantitatively computed and presented in tables. It is worth noting that for increasing the values of the Brinkman-type fluid parameter, the velocity profile decreases. The regression analysis shows that the variation in the velocity for time parameter is statistically significant.
\end{abstract}

\section{Introduction}

Because of its flexible and special properties, fractional calculus has evolved tremendously nowadays. The noninteger derivatives of all the orders are solved utilizing fractional calculus techniques. Fractional calculus is the extension of the classical calculus which has a history of around three centuries. Fractional calculus is a versatile and important method to explain many processes including memories $[1,2]$. In recent years, fractional calculus has been used for many applications in different areas, such as electrochemistry, ground-level water distribution, electromagnetism, elasticity, diffusion, and heat stream conduction [3-5]. For the flow problems, the fractional derivatives approach is used by many researchers. Shah et al. [6] study the viscous fluid in a cylindrical geometry using the Caputo time-fractional derivatives. The solutions were presented in the form special functions. The work was then extended by Ali et al. [7] for the flow of blood. They have considered magnetic particles suspended in the blood and obtained the exact solutions for the flow problem. Caputo fractional derivatives have been used by Vieru et al. [8], Shakeel et al. [9], and Ali et al. [10] for the flow problems, and some interesting and useful results are obtained. Another approach of fractional derivatives is Caputo-Fabrizio fractional derivatives [11] which are also termed as a derivative with nonsingular kernel in the literature. This approach is used by the numerical and analytical solvers for different phenomena in real life. For different situations and analysis, CF derivatives are widely used by Hristov [12], Ali et al. [13], and Fetecau et al. [14]. Abro et al. [15] studied the electrically conducted flow of nanofluid using the concept of CF derivatives and obtained the solutions using special functions. The flow of second-grade fluid with heat transfer and MHD 
effect was analysed by Sheikh et al. [16] using the Caputo-Fabrizio fractional derivatives. The modern concept of the fractional derivatives using the generalized exponential functions (Mittag-Leffler function) has been created in 2016 by Atangana and Baleanu [17]. The kernel of integral associated with that derivative is nonlocal and nonsingular. Atangana and Koca [18] used the modern concept of a fractional derivative to a simple nonlinear system to demonstrate the presence and uniqueness of a solution for the problem. Afterwards, some authors used fractional derivative of Atangana-Baleanu in their study. The theory of frictional derivatives has been used by Sheikh et al. $[19,20]$ for the flow problem of non-Newtonian fluids, which give exact solutions. In these articles, they discussed the difference between the two new fractional operators: Atangana-Baleanu and Caputo-Fabrizio. In both papers, they suggest that the velocity profile obtained for Atangana-Baleanu fractional models falls into a steady-state faster than the velocity profile for CF derivatives.

In the manufacturing and scientific fields, the physical properties of non-Newtonian fluids strongly affect [21-25]. Moreover, in magnetohydrodynamic (MHD) flows, the effects of heat transfer include a wide variety of applications from geosciences to engineering and the chemical sciences field [23, 24, 26-30]. Non-Newtonian fluids demonstrate a complex process which ultimately needs to be described and represented by mathematical modelling. In a porous medium or in clay water contact, this phenomenon of nonNewtonian flow becomes much more complex [23, 24]. In case of flow of non-Newtonian fluid in a porous medium, the effective viscosity becomes nonlinear function of pore velocity and shear flow [30]. The flow velocity behaves inversely under the influence of porosity and magnetohydrodynamics [22]. Due to its huge applications in textile factories, nuclear waste reservoirs, heat pipes, grain storage, and enhanced oil reservoirs storage, the heat and mass transfer through a porous media saturated with fluid has gained great attention. Darcy [31] identified the theoretical analysis and the respective mathematical model of the viscous fluid flow through a medium containing pore. In general, Darcy's implemented law can describe the flows passing over a low permeable region. However, Darcy's law is not effective and applicable to such fluids that move through a medium with high porosity, so Brinkman's model [32] is valid and suitable for such fluids. Extensive research has been done using the Brinkman model on the issue of fluid flow through a porous medium. The properties of the viscous fluid flowing through a porous channel were discussed in [33], using the Brinkman-type fluid model. In two cases, (1) where both walls are porous and (2) where the upper wall is rigid and the lower wall is porous, this problem has been solved. The flow was through the channel, which was highly permeable, and so Brinkman's model was considered. Lin and Payne [34] were analyzing the structural stability of the Brinkman model. Asif et al. [35] addressed the Brinkman-type insight view of fluid flow between two parallel walls. In their study, the oscillatory stress that is applied to the lower plate causes fluid motion. The authors find the exact solutions by using Fourier's mathematical method. They reduced their solutions to already published work by neglecting some of the incorporated parameters to confirm the authenticity of the calculi. Kumar et al. [36] examined Dufour's effect on the thermal-radiating flow of viscous fluid that underlies the highly permeable region. Also, in their work, the authors mention the effects of MHD and chemical reaction. In the light of relative constitutive equations, the governing equations are derived. Some other interesting and relevant studies can be found in [37-42] and the references therein.

In this article, the unsteady MHD flow of Brinkman-type fluid in a vertical channel is considered with heat and mass transfer, keeping in mind the above discussion. Using the Caputo time-fractional derivative principle via generalized Fick's and Fourier's laws, the governing equations are translated into fractional PDEs. The latest transformation is used to transform the energy and concentration equation with initial and boundary conditions. With the joint applications of Laplace and Fourier sine transformation technique, the equations are solved. All the physical conditions are met and can be shown in graphs and tables.

\section{Mathematical Modelling}

We took Brinkman-type fluid motion into consideration in a vertical channel. It is assumed that the flow is in the $x$-axis direction, while the $y$-axis is taken perpendicular to the plates. With ambient temperature $T_{1}$ and ambient concentration $C_{1}$, both the fluid and plates are at rest when $t \leq 0$. At $t=0^{+}$, as seen in Figure 1, the plate at $y=d$ starts to move with velocity $U h(t)$ in its own plane. At $y=d$, the plate temperature and the rate of concentration rose over time $t$ to $\Theta_{1}+\left(\Theta_{2}-\Theta_{1}\right) f(t)$ and $C_{1}+\left(C_{2}-C_{1}\right) g(t)$, respectively.

We suppose the velocity profile as $[22,43]$

$$
\vec{V}=(u, 0,0)
$$

With the aid of the well-known Boussinesq approximation, the free convection flow of fluid of the Brinkmantype, along with the heat and mass transfer, is regulated by the following partial differential equations $[39,40]$ :

$$
\begin{aligned}
\rho \frac{\partial u(y, t)}{\partial t}+\beta_{r} u(y, t)= & \mu \frac{\partial^{2} u(y, t)}{\partial y^{2}}-\sigma B_{0}^{2} u(y, t) \\
& +\rho \beta_{\Theta} g\left(\Theta-\Theta_{1}\right) \\
& +\rho \beta_{C} g\left(C-C_{1}\right), \\
\rho c_{p} \frac{\partial \Theta(y, t)}{\partial t}= & -\frac{\partial q(y, t)}{\partial y}, \\
q(y, t)= & -k \frac{\partial \Theta(y, t)}{\partial y}, \\
\frac{\partial C(y, t)}{\partial t}= & -\frac{\partial j(y, t)}{\partial y},
\end{aligned}
$$




$$
j(y, t)=-D \frac{\partial C(y, t)}{\partial y}
$$

with the initial and boundary conditions

$$
\left.\begin{array}{lll}
u(y, 0)=0, & \Theta(y, 0)=\Theta_{1}, & C(y, 0)=C_{1}, \\
u(0, t)=0, & \Theta(0, t)=\Theta_{1}, & C(0, t)=C_{1}, \\
u(d, t)=U h(t), & \Theta(d, t)=\Theta_{1}+\left(\Theta_{2}-\Theta_{1}\right) f(t), & C(d, t)=C_{1}+\left(C_{2}-C_{1}\right) g(t),
\end{array}\right\},
$$

$$
\frac{\partial v(\xi, \tau)}{\partial \tau}=\frac{\partial^{2} v(\xi, \tau)}{\partial \xi^{2}}-A v(\xi, \tau)+\operatorname{Gr} \theta(\xi, \tau)+\operatorname{Gm} \Phi(\xi, \tau),
$$

viscosity, density, fluid velocity, material parameter, temperature, thermal expansion coefficient, coefficient of concentration, acceleration due to gravity, specific heat capacity, thermal conductivity, and mass diffusivity, respectively.

Introducing the following dimensionless variables

$$
\begin{aligned}
v & =\frac{u}{U}, \\
\xi & =\frac{y}{d}, \\
\tau & =\frac{v}{d^{2}} t, \\
\theta & =\frac{\Theta-\Theta_{1}}{\Theta_{2}-\Theta_{1}}, \\
\Phi & =\frac{C-C_{1}}{C_{2}-C_{1}}, \\
\delta & =\frac{q d}{k\left(\Theta_{2}-\Theta_{1}\right)}, \\
\lambda & =\frac{j d}{D\left(C_{2}-C_{1}\right)}, \\
f(\tau) & =f\left(\frac{d^{2}}{v} t\right), \\
g(\tau) & =g\left(\frac{d^{2}}{v} t\right), \\
h(\tau) & =h\left(\frac{d^{2}}{v} t\right) .
\end{aligned}
$$

$$
\frac{\partial \theta(\xi, \tau)}{\partial \tau}=-\frac{1}{\operatorname{Pr}} \frac{\partial \delta(\xi, \tau)}{\partial \xi}
$$

$$
\delta(\xi, \tau)=-\frac{\partial \theta(\xi, \tau)}{\partial \xi},
$$

$\frac{\partial \Phi(\xi, \tau)}{\partial \tau}=-\frac{1}{\mathrm{Sc}} \frac{\partial \lambda(\xi, \tau)}{\partial \xi}$,

$$
\lambda(\xi, \tau)=-\frac{\partial \Phi(\xi, \tau)}{\partial \xi}
$$

$$
\left.\begin{array}{l}
v(\xi, 0)=0 \\
v(0, \tau)=0 \\
v(1, \tau)=h(\tau) \\
\theta(\xi, 0)=0 \\
\theta(0, \tau)=0 \\
\theta(1, \tau)=f(\tau) \\
\Phi(\xi, 0)=0 \\
\Phi(0, \tau)=0 \\
\Phi(1, \tau)=g(\tau)
\end{array}\right\}
$$

into equations (2)-(6), we get

where $\mathrm{Gr}=\left(g d^{2} \beta_{\Theta} / \nu U\right)\left(\Theta_{2}-\Theta_{1}\right)$ is the thermal Grashof number, $\mathrm{Gm}=\left(g d^{2} \beta_{C} / \nu U\right)\left(C_{2}-C_{1}\right)$ is the mass Grashof number, $\gamma_{B}=\left(\beta_{r} d^{2} / v\right)$ is the Brinkman-type fluid parameter, $M=\left(\sigma B_{0}^{2} d^{2} / \mu\right)$ is Hartman number, $\operatorname{Pr}=\left(\mu c_{p} / k\right)$ is the Prandtl number, $\mathrm{Sc}=(\nu / D)$ is the Schmidt number, and $A=M+\gamma_{B}$. 


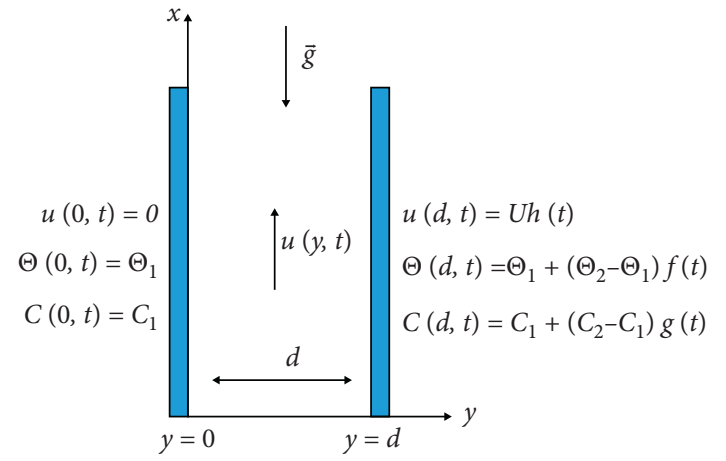

Figure 1: Geometry of the flow.

\section{Fractional Model}

The generalized laws of Fourier and Fick are utilized as follows to establish a fractional model for the convective part of the referred flow problem:

$$
\begin{array}{cl}
\delta(\xi, \tau)=-{ }^{C} \wp_{\tau}^{1-\alpha}\left(\frac{\partial \theta(\xi, \tau)}{\partial \xi}\right) ; & 0<\alpha \leq 1, \\
\lambda(\xi, \tau)=-{ }^{C} \wp_{\tau}^{1-\alpha}\left(\frac{\partial \Phi(\xi, \tau)}{\partial \xi}\right) ; & 0<\alpha \leq 1,
\end{array}
$$

where ${ }^{C} \wp_{\tau}^{\alpha}(\cdot)$ is the time-fractional operator developed by Caputo [44] and is described as [7]

$$
\begin{aligned}
{ }_{\wp_{\tau}}^{\alpha} r(y, t) & =\frac{1}{\Gamma(1-\alpha)} \int_{0}^{t} \dot{r}(y, s)(t-s)^{-\alpha} \mathrm{d} s \\
& =\eta_{\alpha}(t) * \dot{r}(y, t) ; \quad 0<\alpha \leq 1 .
\end{aligned}
$$

Here, $\eta_{\alpha}(t)=\left(t^{-\alpha} / \Gamma(1-\alpha)\right)$ is the singular power-law kernel.

Furthermore,

$$
\begin{gathered}
L\left\{\eta_{\alpha}(t)\right\}=\frac{1}{s^{1-\alpha}}, \\
\left\{\eta_{1-\alpha} * \eta_{\alpha}\right\}(t)=1, \\
\eta_{0}(t)=L^{-1}\left\{\frac{1}{s}\right\}=1, \\
\eta_{1}(t)=L^{-1}\{1\}=\zeta(t),
\end{gathered}
$$

where $L\{\cdot\}$ is the Laplace transform, $\zeta(\cdot)$ is Dirac's delta function, and $s$ is the Laplace transform parameter.

Using the above properties and second form equation (17), it is convenient to show that

$$
\begin{aligned}
& { }_{{ }^{C}}{ }_{\tau}^{0} r(y, t)=r(y, t)-r(y, 0), \\
& { }^{C}{ }_{{ }_{\tau}}^{1} r(y, t)=\frac{\partial r(y, t)}{\partial t} .
\end{aligned}
$$

Utilizing the definition of Caputo time-fractional operator form equation (23) and using equations (10, 12, 15, and 16), we arrived at

$$
\begin{gathered}
\frac{\partial \theta(\xi, \tau)}{\partial t}=\frac{1_{C}}{\operatorname{Pr}} \wp_{\tau}^{1-\alpha}\left(\frac{\partial^{2} \theta(\xi, \tau)}{\partial^{2} \xi}\right), \\
\frac{\partial \Phi(\xi, \tau)}{\partial t}=\frac{1_{C}}{\operatorname{Sc}_{C}} \wp_{\tau}^{1-\alpha}\left(\frac{\partial^{2} \Phi(\xi, \tau)}{\partial^{2} \xi}\right) .
\end{gathered}
$$

We recalled the time-fractional integral operator to get the finest form for the last two equations:

$$
\mathfrak{\Im}_{t}^{\alpha} r(y, t)=\left(\eta_{1-\alpha} * r\right)(t)=\frac{1}{\Gamma(\alpha)} \int_{0}^{t} r(y, s)(t-s)^{\alpha-1} \mathrm{~d} s .
$$

This is the inverse operator of the derivative operator ${ }^{C} \wp_{\tau}^{\alpha}(\cdot)$. Using the properties from equation (18), we have

$$
\begin{aligned}
\left(\mathfrak{\Im}_{t}^{\alpha}{ }_{0}{ }^{C} \wp_{\tau}^{\alpha}\right) r(y, t) & =\Im_{t}^{\alpha}\left({ }^{C} \wp_{\tau}^{\alpha} r(y, t)\right)=\left[\eta_{1-\alpha} *\left(\eta_{\alpha} * \dot{r}\right)\right](t) \\
& =\left[\left(\eta_{1-\alpha} * \eta_{\alpha}\right) * \dot{r}\right] \dot{r}(t)=[1 * \dot{r}](t) \\
& =r(y, t)-r(y, 0),
\end{aligned}
$$

$$
\Rightarrow\left(\mathfrak{\Im}_{t}^{\alpha}{ }^{C}{ }^{C} \wp_{\tau}^{\alpha}\right) r(y, t)=r(y, t), \quad \text { if } r(y, 0)=0 .
$$

Using the property $\mathfrak{\Im}_{t}^{1-\alpha} \dot{r}(y, t)=\left(\eta_{\alpha} * \dot{r}\right)$ $(t)={ }^{C} \wp_{\tau}^{\alpha} r(y, t)$, equations (20) and (21) can be written as

$$
\begin{gathered}
{ }_{\wp_{\tau}^{\alpha} \theta(\xi, \tau)}^{\alpha}=\frac{1}{\operatorname{Pr}} \frac{\partial^{2} \theta(\xi, \tau)}{\partial^{2} \xi}, \\
{ }_{\wp_{\tau}{ }_{\tau}^{\alpha} \Phi(\xi, \tau)}=\frac{1}{\operatorname{Sc}} \frac{\partial^{2} \Phi(\xi, \tau)}{\partial^{2} \xi},
\end{gathered}
$$

\section{Solution of the Problem}

4.1. Energy Field. The following transformation is used:

$$
\chi(\xi, \tau)=\theta(\xi, \tau)-\xi f(\tau) .
$$

Equation (25) takes the following form:

$$
{ }^{C} \wp_{\tau}^{\alpha} \chi(\xi, \tau)-\xi^{C}{ }_{\gamma_{\tau}}^{\alpha} f(\tau)=\frac{1}{\operatorname{Pr}} \frac{\partial^{2} \chi(\xi, \tau)}{\partial^{2} \xi} .
$$

With the corresponding initial and boundary conditions,

$$
\begin{aligned}
& \chi(\xi, 0)=0, \\
& \chi(0, \tau)=0, \\
& \chi(1, \tau)=0 .
\end{aligned}
$$

Applying the Laplace and Fourier sine transform, we get

$$
\bar{\chi}_{F}(n, s)=s \bar{f}(s) \frac{(-1)^{n}}{n \pi} \frac{s^{\alpha-1}}{s^{\alpha}+\left((n \pi)^{2} / \operatorname{Pr}\right)} .
$$

Inverting the integral transformations of equation (30), we have 
$\chi(\xi, \tau)=2 \sum_{n=1}^{\infty} \frac{(-1)^{n} \sin (\xi n \pi)}{n \pi} \int_{0}^{\tau} \dot{f}(\tau-t) E_{\alpha, \alpha-1}\left(-\frac{(n \pi)^{2}}{\operatorname{Pr}} t^{\alpha}\right) \mathrm{d} t$.

Therefore, the solution of the energy equation is

$$
\theta(\xi, \tau)=\chi(\xi, \tau)+\xi f(\tau)
$$

4.2. Concentration Field. The following transformation is used:

$$
\Psi(\xi, \tau)=\Phi(\xi, \tau)-\xi g(\tau) .
$$

Equation (26) takes the following form:

$$
{ }^{C} \wp_{\tau}^{\alpha} \Psi(\xi, \tau)-\xi^{C} \wp_{\tau}^{\alpha} g(\tau)=\frac{1}{\mathrm{Sc}} \frac{\partial^{2} \Psi(\xi, \tau)}{\partial^{2} \xi} .
$$

With the corresponding initial and boundary conditions,

$$
\begin{aligned}
& \Psi(\xi, 0)=0, \\
& \Psi(0, \tau)=0, \\
& \Psi(1, \tau)=0 .
\end{aligned}
$$

Applying the Laplace and Fourier sine transform, we get

$$
\bar{\Psi}_{F}(n, s)=s \bar{g}(s) \frac{(-1)^{n}}{n \pi} \frac{s^{\alpha-1}}{s^{\alpha}+\left((n \pi)^{2} / \mathrm{Sc}\right)} .
$$

Inverting the integral transformations of equation (36), we have

$\Psi(\xi, \tau)=2 \sum_{n=1}^{\infty} \frac{(-1)^{n} \sin (\xi n \pi)}{n \pi} \int_{0}^{\tau} \dot{g}(\tau-t) E_{\alpha, \alpha-1}\left(-\frac{(n \pi)^{2}}{S c} t^{\alpha}\right) \mathrm{d} t$.
The final solution for the concentration equation is

$$
\Phi(\xi, \tau)=\Psi(\xi, \tau)+\xi g(\tau)
$$

4.3. Velocity Profile. Applying the Laplace and Fourier transform to equation (9) using equation (14), we arrived at

$$
\begin{aligned}
\bar{v}_{F}(n, s)= & \frac{(-1)^{n+1} \bar{h}(s)}{n \pi}+\left(\frac{\mathfrak{R}_{5}}{s}+\frac{\mathfrak{R}_{6}}{s+\mathfrak{R}_{1}}\right) \frac{(-1)^{n} s \bar{h}(s)}{n \pi} \\
& +\frac{\mathrm{Gr} \bar{\theta}_{F}(n, s)}{\left(s+\mathfrak{R}_{1}\right)}+\frac{\mathrm{Gm} \bar{\Phi}_{F}(n, s)}{\left(s+\mathfrak{R}_{1}\right)},
\end{aligned}
$$

where

$$
\begin{aligned}
& \mathfrak{R}_{1}=A+(n \pi)^{2}, \\
& \mathfrak{R}_{2}=\mathfrak{R}_{1}(n \pi)^{2}, \\
& \mathfrak{R}_{3}=1-\frac{(n \pi)^{2}}{\mathfrak{R}_{1}}, \\
& \mathfrak{R}_{4}=\mathfrak{R}_{1}-(n \pi)^{2}, \\
& \mathfrak{R}_{5}=\frac{\mathfrak{R}_{4}}{\mathfrak{R}_{1}}, \\
& \mathfrak{R}_{6}=\frac{\left(\mathfrak{R}_{3} \mathfrak{R}_{1}+\mathfrak{R}_{2} \mathfrak{R}_{1}+\mathfrak{R}_{4}\right)}{\mathfrak{R}_{1}} .
\end{aligned}
$$

Inverting the Laplace and Fourier sine transformations, the final solution is

$$
\begin{aligned}
v(\xi, \tau)= & h(\tau) \xi+2 \sum_{n=1}^{\infty} \frac{(-1)^{n}}{n \pi} \dot{h}(\tau) *\left(\Re_{5} H(\tau)+\mathfrak{R}_{6} \exp \left(-\mathfrak{R}_{1} \tau\right)\right) \sin (\xi n \pi) \\
& +2 \frac{G r}{\Re} \sum_{n=1}^{\infty}\left(\frac{(-1)^{n}}{n \pi} \exp \left(-\Re_{1} \tau\right) *\left(\int_{0}^{\tau} \dot{f}(\tau-t) E_{\alpha, \alpha-1}\left(-\frac{(n \pi)^{2}}{\operatorname{Pr}} t^{\alpha}\right) \mathrm{d} t+f(\tau)\right)\right) \sin (\xi n \pi) \\
& +2 \frac{G m}{\mathfrak{R}} \sum_{n=1}^{\infty}\left(\frac{(-1)^{n}}{n \pi} \exp \left(-\mathfrak{R}_{1} \tau\right) *\left(\int_{0}^{\tau} \dot{g}(\tau-t) E_{\alpha, \alpha-1}\left(-\frac{(n \pi)^{2}}{\mathrm{Sc}} t^{\alpha}\right) \mathrm{d} t+g(\tau)\right)\right) \sin (\xi n \pi),
\end{aligned}
$$


where $H(\tau)$ is the unit step function and $E_{a, b}(\cdot)$ is the Mittag-Leffler function [45].

\section{Special Cases}

5.1. Flow in the Absence of Mass Transfer. In the absence of mass concentration $(\mathrm{Gm}=0)$, equation (41) takes the following form:

$$
\begin{aligned}
v(\xi, \tau)= & h(\tau) \xi+2 \sum_{n=1}^{\infty} \frac{(-1)^{n}}{n \pi} \dot{h}(\tau) *\left(\mathfrak{R}_{5} H(\tau)+\mathfrak{R}_{6} \exp \left(-\mathfrak{R}_{1} \tau\right)\right) \sin (\xi n \pi) \\
& +2 \frac{G r}{\mathfrak{R}} \sum_{n=1}^{\infty}\left(\frac{(-1)^{n}}{n \pi} \exp \left(-\mathfrak{R}_{1} \tau\right) *\left(\int_{0}^{\tau} \dot{f}(\tau-t) E_{\alpha, \alpha-1}\left(-\frac{(n \pi)^{2}}{\operatorname{Pr}} t^{\alpha}\right) \mathrm{d} t+f(\tau)\right)\right) \sin (\xi n \pi)
\end{aligned}
$$

\section{Entropy Generation}

For the flow of Brinkman-type fluid with the magnetic field and in the absence of mass concentration, taking into account the velocity from equation (42), the entropy generation is defined by [46]

$$
S_{G}=\underbrace{\frac{k}{\Theta_{1}^{2}}\left\{\frac{\partial \Theta(y, t)}{\partial y}\right\}^{2}}_{\begin{array}{c}
\text { entropy generation } \\
\text { due to heat transfer }
\end{array}}+\underbrace{\frac{\mu}{\Theta_{1}}\left\{\frac{\partial u(y, t)}{\partial y}\right\}^{2}+\frac{\beta_{r}}{\Theta_{1}} u^{2}(y, t)}_{\text {entropy generation }}+\underbrace{\frac{\sigma B_{0}^{2}}{\Theta_{1}} u^{2}(y, t)}_{\text {due to fluid friction }} .
$$

The dimensionless form of equations (43) is given as

$$
\begin{aligned}
N s(\xi, \tau)=\frac{S_{G}}{S_{0}}= & \left\{\frac{\partial \theta(\xi, \tau)}{\partial \xi}\right\}^{2}+\frac{\operatorname{Br}}{\Omega}\left\{\frac{\partial v(\xi, \tau)}{\partial \xi}\right\}^{2} \\
& +\frac{\operatorname{Br} \gamma_{B}}{\Omega} v^{2}(\xi, \tau)+\frac{\operatorname{Br} M}{\Omega} v^{2}(\xi, \tau),
\end{aligned}
$$

with $S_{0}=\left(k U\left(\Theta_{2}-\Theta_{1}\right) / \nu^{2} \Theta_{1}^{2}\right), \mathrm{Br}=\left(U^{2} \mu / k\left(\Theta_{2}-\Theta_{1}\right)\right)$, $\Omega=\left(\Theta_{2}-\Theta_{1} / \Theta_{1}\right)$ which are the characteristics of entropy generation, Brinkman number, and dimensionless temperature difference, respectively.

6.1. The Bejan Number. The Bejan number is defined as the irreversibility distribution parameter mathematically:

$$
\mathrm{Be}=\frac{\text { entropy generation due to heat transfer }}{\text { total entropy generation }} \text {. }
$$

\section{Nusselt Number}

Nusselt number is an important physical quantity specially for engineers and industrialists. In nondimensional form, Nusselt number is given by

$$
\mathrm{Nu}=\left.\frac{\partial \theta(\xi, \tau)}{\partial \xi}\right|_{\xi=1} .
$$

\section{Sherwood Number}

The gradient of mass concentration is termed as Sherwood number. In nondimensional form, Sherwood number is given by

$$
\mathrm{Sh}=\left.\frac{\partial \Phi(\xi, \tau)}{\partial \xi}\right|_{\xi=1}
$$

\section{Results and Discussion}

In this analysis, we address the unsteady flow of Brinkmantype fluid in a vertical channel. The cumulative effects of heat and mass transfer have been considered. A fractional model was developed using the generalized Fick's and Fourier's laws. In order to convert the equation into simpler form, a new transformation is applied, and then the Laplace and Fourier sine transformations are used to obtain solutions in closed form. Figures and tables demonstrated the effect of different embedded parameters on the velocity, temperature, and concentration profiles. Regression analysis is introduced to estimate the time-based velocity profile.

The influence of the fractional parameter $\alpha$ is shown in Figure 2. From this figure, the fluid velocity is the increasing function of fractional parameter. Figures 3 and 4 are sketched to depict the influence of thermal and mass Grashof numbers. These figures show that the velocity is increasing with the increasing values of $\mathrm{Gr}$ and $\mathrm{Gm}$. This is due to buoyancy forces, these forces become dominant with the increasing values of Grand Gm, and as a result, the boundary 


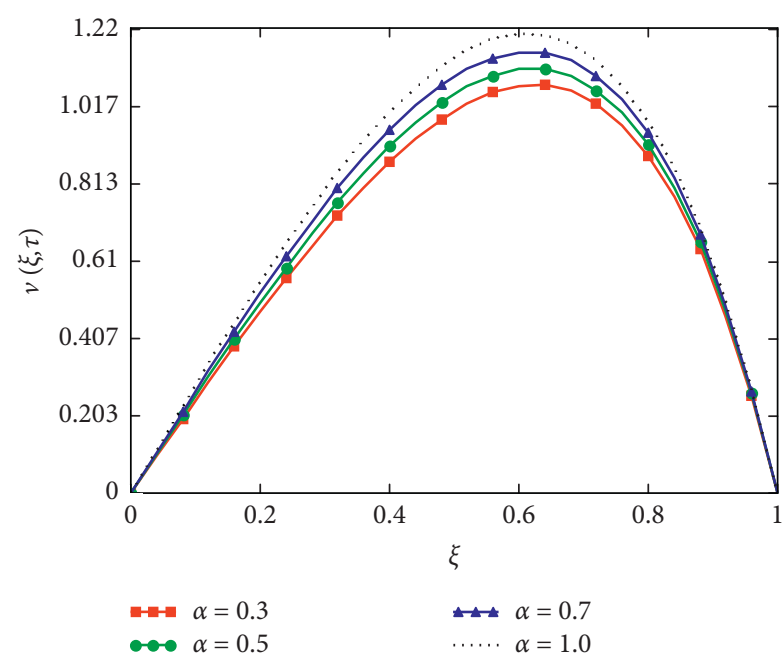

FIGURE 2: Variations in velocity profile against $\xi$ if $h(\tau)=0$ for different values of $\alpha$.

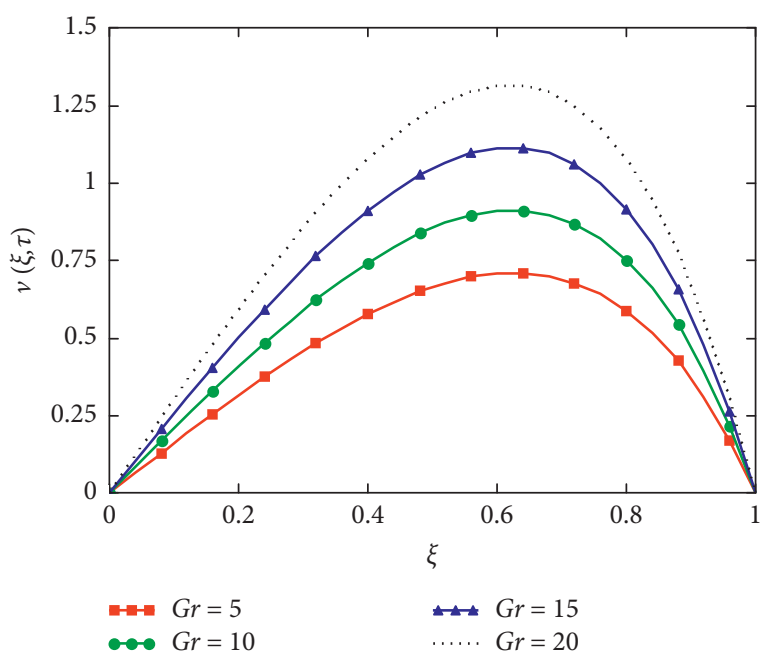

FIGURE 3: Variations in velocity profile against $\xi$ if $h(\tau)=0$ for different values of Gr.

layer thickness decreases. Both the figures show that imposed boundary conditions are satisfied. Prandtl number is the ration of viscous forces to the thermal forces. The increasing values of Prandtl number mean that the viscous forces become dominant over the thermal forces, and as a result, the fluid velocity decreases as shown in Figure 5. The variations in velocity profile due to change in Brinkman-type fluid parameter are depicted in Figure 6. From this figure, interesting results are obtained. The velocity is decreasing with increasing values of the material parameter $\gamma_{B}$. Figure 7 is plotted in order to show the influence of Schmidt number on the fluid flow. It is noticed that the fluid velocity is decelerating with the increasing values of Sc. The influence of Hartman number is depicted in Figure 8. Velocity of the fluid is decreasing with the increasing values of $M$. As the increasing values of $M$ strengthen the Lorentz forces which are the flow opposing forces. In order to show the validation

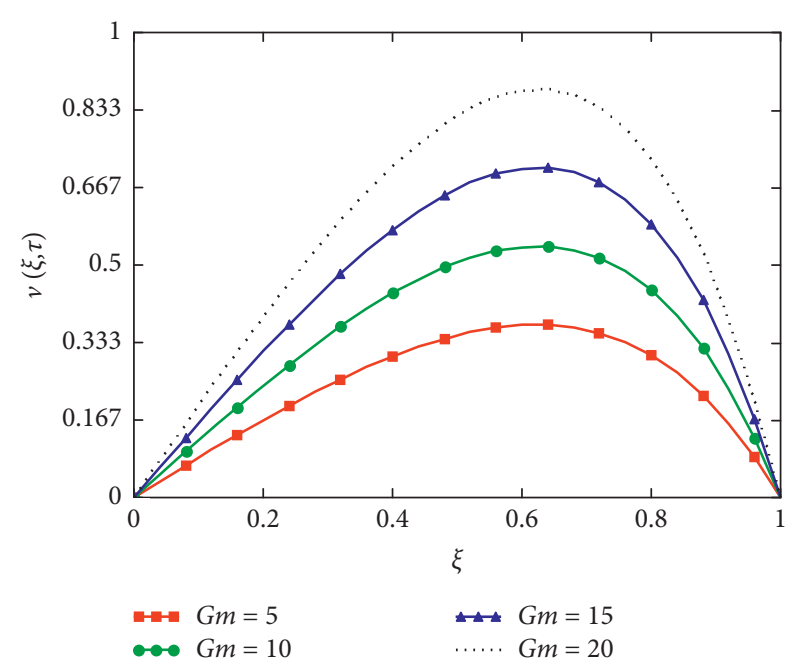

FIGURE 4: Variations in velocity profile against $\xi$ if $h(\tau)=0$ for different values of $M$.

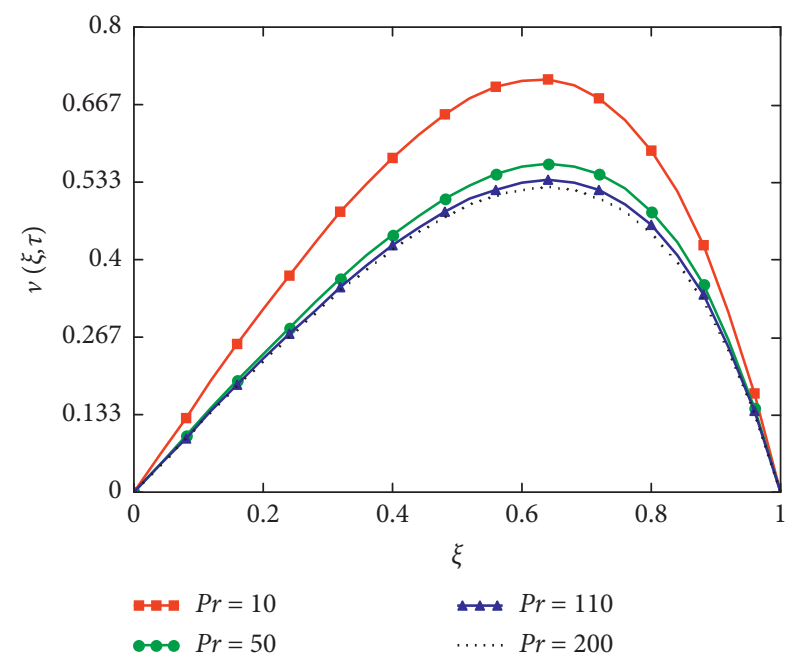

FIGURE 5: Variations in velocity profile against $\xi$ if $h(\tau)=0$ for different values of Pr.

of the obtained solution, Figure 9 is plotted. In this figure, $h(\tau)=\tau(\tau=0.5,1,1.5$ and 2$)$ is taken and the conditions are satisfied. This means that for all type of boundary conditions, the obtained solutions are valid.

The influence of fractional parameter on the temperature profile is shown in Figure 10. The figure shows the temperature-increasing function of the fractional parameter. The effect of Prandtl number on the temperature profile is shown in Figure 11. The temperature is decreasing with the increasing values of Prandtl number, because the thermal forces weaken with the increasing values of Prandtl number. Concentration profile is plotted for various values of the fractional parameter in Figure 12. The same behavior is noticed as in the case of temperature in this figure. The concentration of mass is decreasing with the increasing values of Schmidt number which is shown in Figure 13. The influence of the Brinkman-type fluid parameter on the 


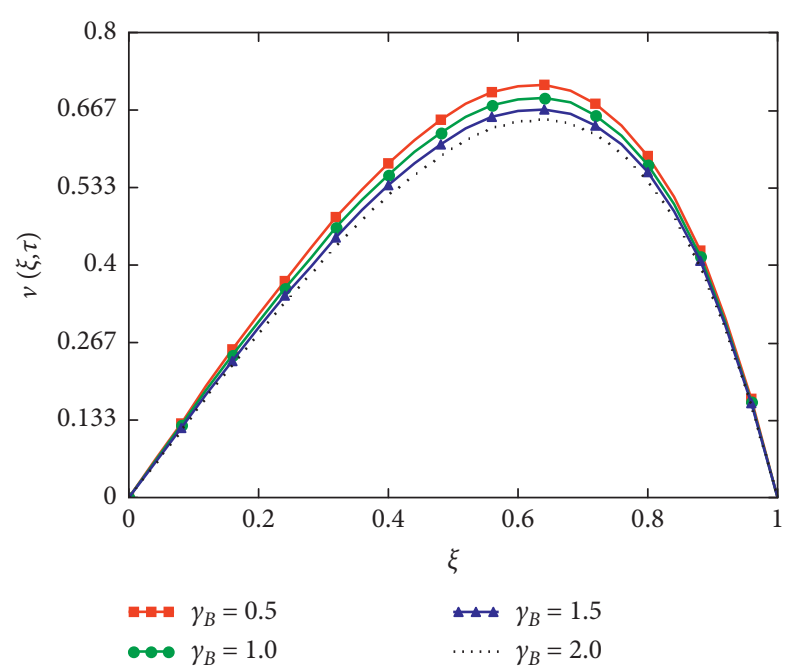

FIGURE 6: Variations in velocity profile against $\xi$ if $h(\tau)=0$ for different values of $\gamma_{B}$.

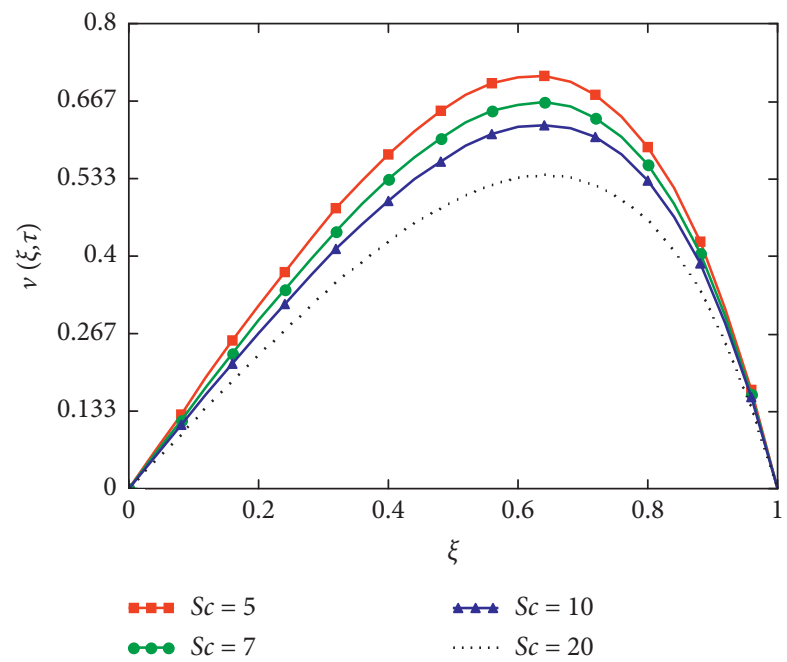

FIGURE 7: Variations in velocity profile against $\xi$ if $h(\tau)=0$ for different values of Sc.

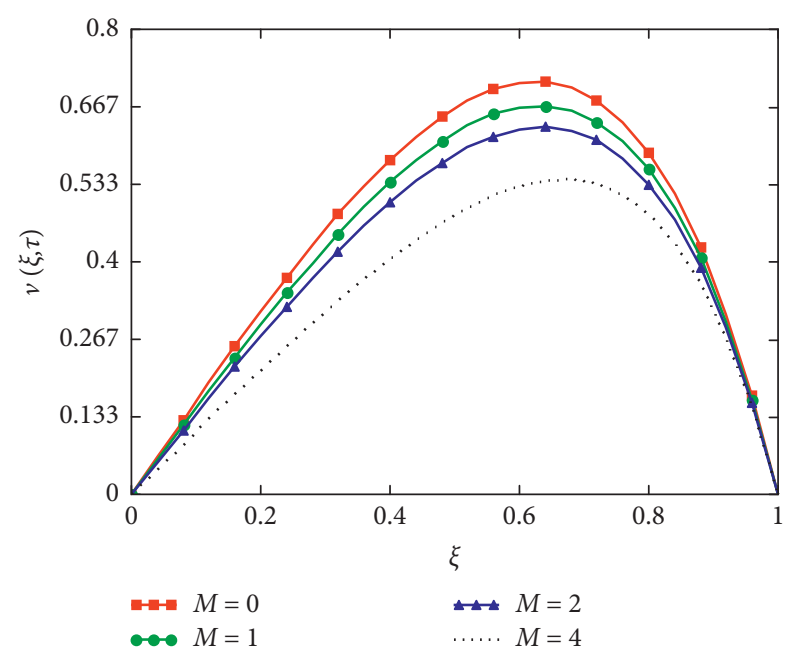

FIGURE 8: Variations in velocity profile against $\xi$ if $h(\tau)=0$ for different values of $M$.

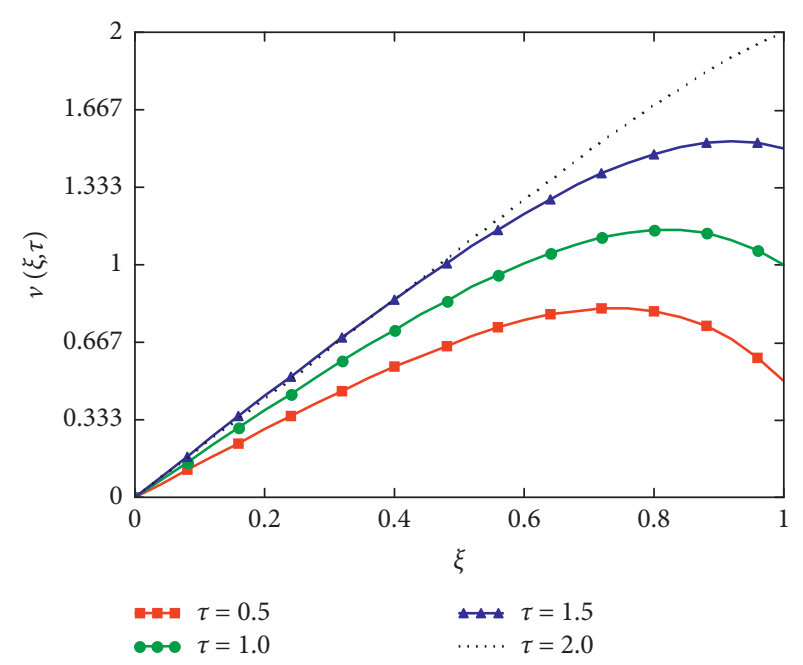

FIgURE 9: Variations in velocity profile against $\xi$ if $h(\tau)=\tau$ for different values of $\tau$.

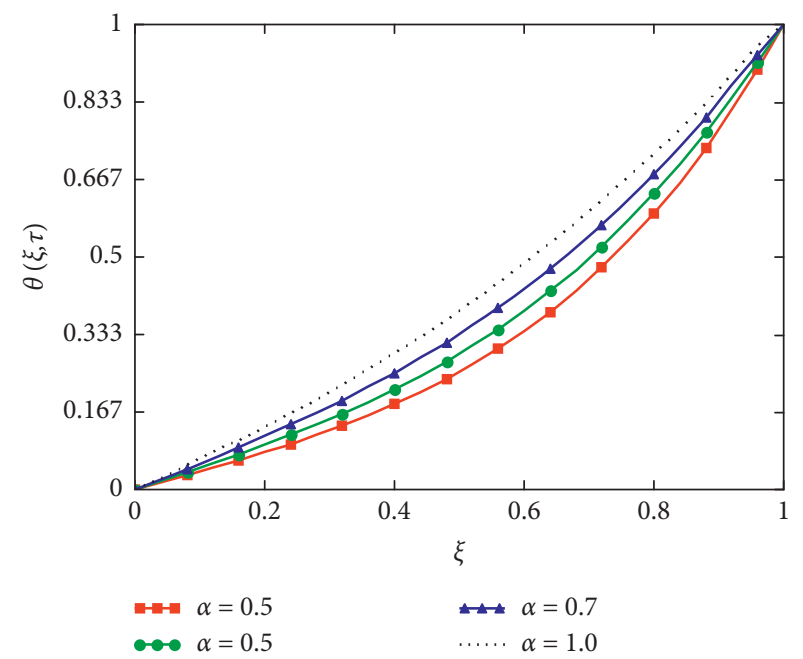

FIgURE 10: Variations in temperature profile against $\xi$ for different values of $\alpha$.

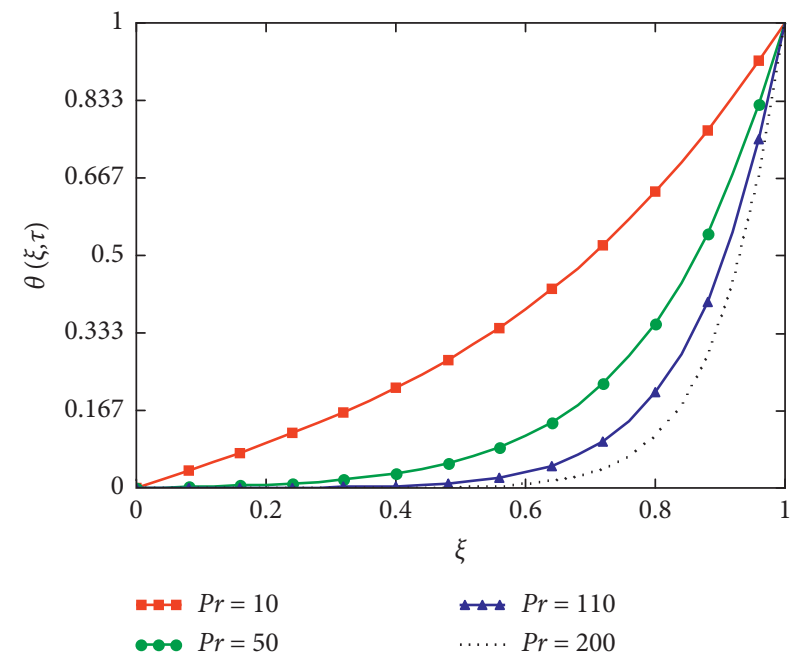

FIGURE 11: Variations in temperature profile against $\xi$ for different values of Pr. 


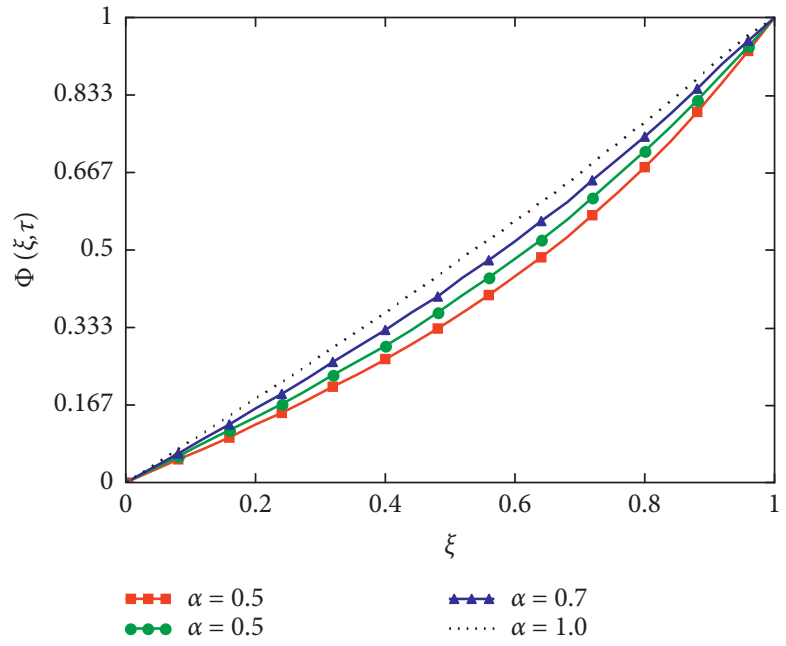

FIGURE 12: Variations in Concentration profile against $\xi$ for different values of $\alpha$.

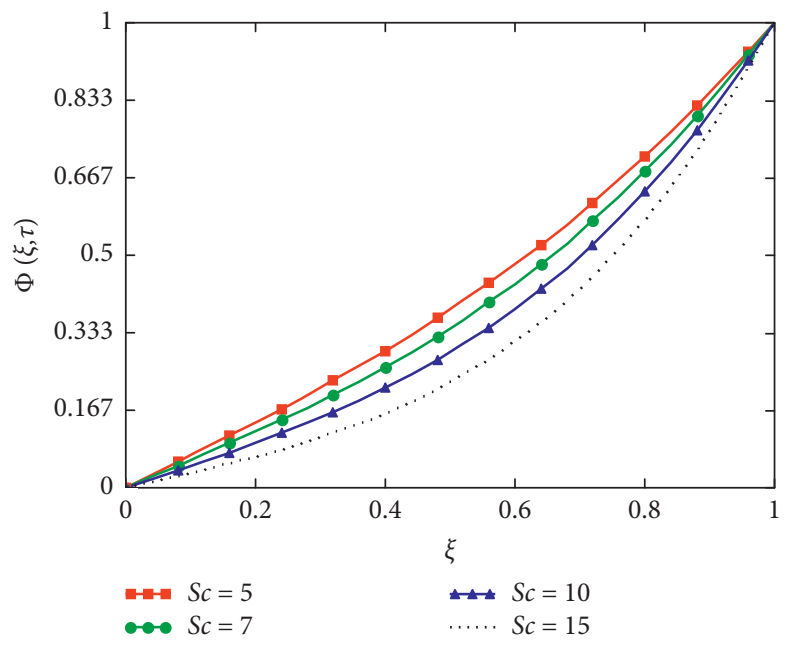

FIgURE 13: Variations in Concentration profile against $\xi$ for different values of Sc.

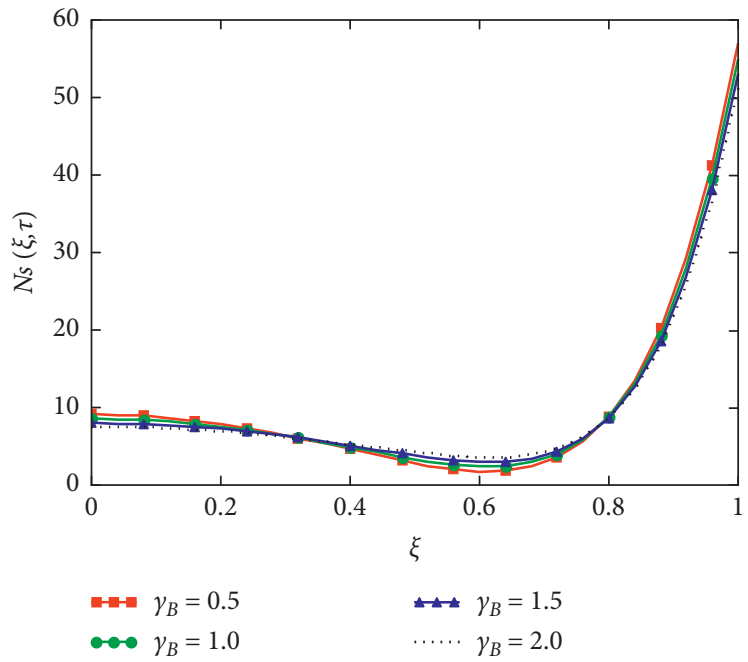

FIGURE 14: Variations Entropy Generation against $\xi$ for different values of $\gamma_{B}$. 


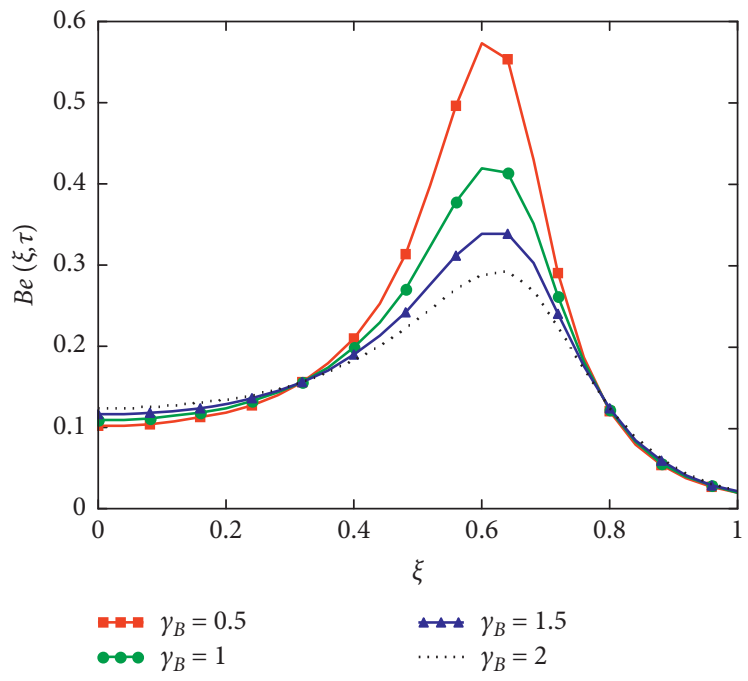

Figure 15: Variations Bejan Number against $\xi$ for different values of $\gamma_{B}$.

TABLE 1: Variations in velocity profile against $\xi$ if $h(\tau)=0$ for different values of $\tau$.

\begin{tabular}{|c|c|c|c|c|}
\hline$\xi$ & $v(\xi, \tau)$ at $\tau=0.5$ & $v(\xi, \tau)$ at $\tau=1$ & $v(\xi, \tau)$ at $\tau=1.5$ & $v(\xi, \tau)$ at $\tau=2$ \\
\hline 0.04 & 0.089 & 0.094 & 0.096 & 0.098 \\
\hline 0.08 & 0.178 & 0.188 & 0.192 & 0.195 \\
\hline 0.12 & 0.266 & 0.28 & 0.286 & 0.29 \\
\hline 0.16 & 0.352 & 0.37 & 0.379 & 0.384 \\
\hline 0.2 & 0.436 & 0.459 & 0.469 & 0.476 \\
\hline 0.24 & 0.518 & 0.544 & 0.556 & 0.564 \\
\hline 0.28 & 0.596 & 0.625 & 0.639 & 0.649 \\
\hline 0.32 & 0.67 & 0.702 & 0.718 & 0.728 \\
\hline 0.36 & 0.739 & 0.774 & 0.791 & 0.802 \\
\hline 0.4 & 0.802 & 0.839 & 0.858 & 0.87 \\
\hline 0.44 & 0.858 & 0.898 & 0.917 & 0.929 \\
\hline 0.48 & 0.907 & 0.948 & 0.967 & 0.981 \\
\hline 0.52 & 0.947 & 0.988 & 1.008 & 1.022 \\
\hline 0.56 & 0.976 & 1.017 & 1.038 & 1.051 \\
\hline 0.6 & 0.993 & 1.034 & 1.054 & 1.068 \\
\hline 0.64 & 0.997 & 1.036 & 1.056 & 1.07 \\
\hline 0.68 & 0.985 & 1.022 & 1.042 & 1.055 \\
\hline 0.72 & 0.955 & 0.99 & 1.008 & 1.021 \\
\hline 0.76 & 0.905 & 0.937 & 0.954 & 0.965 \\
\hline 0.8 & 0.833 & 0.861 & 0.875 & 0.885 \\
\hline 0.84 & 0.734 & 0.758 & 0.77 & 0.778 \\
\hline 0.88 & 0.605 & 0.624 & 0.633 & 0.64 \\
\hline 0.92 & 0.443 & 0.456 & 0.463 & 0.467 \\
\hline 0.96 & 0.243 & 0.25 & 0.253 & 0.255 \\
\hline 1 & 0 & 0 & 0 & 0 \\
\hline
\end{tabular}

entropy generation is shown in Figure 14. It is revealed from this figure that near the plates, the entropy generation decreases with increasing vales of the Brinkman-type fluid parameter, while the entropy generation increases in the middle of the channel for increasing values of $\gamma_{B}$. The impact of $\gamma_{B}$ on the Bejan number is shown in Figure 15. From this figure, it is clear that the Bejan number increases near the walls of the channel while decreases at the middle of the channel for increasing vales of $\gamma_{B}$. Table 1 is presented to show the influence of the dimensionless time parameter on the fluid velocity, the velocity is showing an increasing trend for higher values of $\tau$. The variation in the velocity and the predicted velocity against $\tau$ is presented in Figure 16 and Table 2 for fixed value of $\xi$, which shows that velocity is directly related to $\tau$. This variation is statistically significant [47] as the $P$ value is less than 0.05 as shown in Table 3 . 


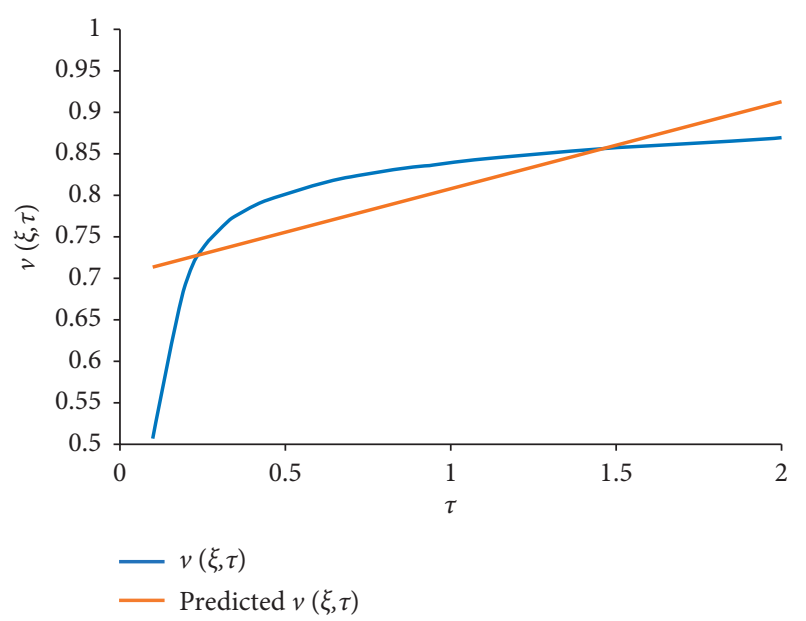

FIGURE 16: The prediction of velocity for increasing time at $\xi=0.4$.

TABLE 2: Variation in velocity against $\tau$.

\begin{tabular}{lccccccccccc}
\hline$\tau$ & 0 & 0.2 & 0.4 & 0.6 & 0.8 & 1 & 1.2 & 1.4 & 1.6 & 1.8 & 2 \\
\hline$v(\xi, \tau)$ & 0 & 0.7 & 0.79 & 0.81 & 0.83 & 0.84 & 0.85 & 0.86 & 0.9 & 0.87 & 0.9
\end{tabular}

TABLE 3: Regression table of velocity for $\tau$.

\begin{tabular}{lcc}
\hline & Coefficients & $P$ value \\
\hline Intercept & 0.704832 & $9.26 \mathrm{E}-16$ \\
$\tau$ & 0.103541 & 0.000225 \\
\hline
\end{tabular}

\section{Conclusion}

The fractional Brinkman-type fluid model is developed using a new methodology in this research. The generalized Fick and Fourier laws are used to develop a fractional model. The Laplace and Fourier transformation methods are used to solve the problem. The results produced are drawn up and displayed in tables. The main findings of this analysis are as follows:

(1) The new transformation is more reliable for the solution of the fractional model. It is easier to solve the fractional model using this transformation.

(2) This transformation is reducing the computational time for finding the exact solutions of such problems.

(3) The velocity reduces with higher values of Hartman's number and the Brinkman-type fluid parameter.

(4) For various values of $\alpha$, variations in all the three profiles are shown. It is important to note here that for a fixed value of time, we have different lines in the graph. This result demonstrates the fluid's memory effect, which cannot be seen from the integer order model.

(5) The variation in the velocity profile for different values of the fractional parameter is statistically significant.
(6) The entropy generation and Bejan number show different behaviors for the Brinkman-type fluid parameter near the walls and in the middle of the channel.

\section{Data Availability}

The data used to support the findings of this study are included in this paper and available without any restriction.

\section{Conflicts of Interest}

The authors declare that they have no conflicts of interest.

\section{References}

[1] A. Atangana and B. Alkahtani, "Analysis of the Keller-Segel model with a fractional derivative without singular kernel," Entropy, vol. 17, no. 6, pp. 4439-4453, 2015.

[2] J. F. Gómez-Aguilar, A. Atangana, and V. F. Morales-Delgado, "Electrical circuits RC, LC, and RL described by Atangana-Baleanu fractional derivatives," International Journal of Circuit Theory and Applications, vol. 45, no. 11, pp. 1514-1533, 2017.

[3] H. Singh, "Analysis for fractional dynamics of Ebola virus model," Chaos, Solitons \& Fractals, vol. 138, Article ID 109992, 2020.

[4] K. A. Abro, I. Khan, and A. Tassaddiq, "Application of Atangana-Baleanu fractional derivative to convection flow of MHD Maxwell fluid in a porous medium over a vertical plate," Mathematical Modelling of Natural Phenomena, vol. 13, no. 1, p. 1, 2018.

[5] I. Khan, M. Saqib, M. Saqib, and A. M. Alqahtani, "Channel flow of fractionalized H2O-based CNTs nanofluids with Newtonian heating," Discrete \& Continuous Dynamical Systems-S, vol. 13, no. 3, pp. 769-779, 2020.

[6] N. A. Shah, D. Vieru, and C. Fetecau, "Effects of the fractional order and magnetic field on the blood flow in cylindrical domains," Journal of Magnetism Magnetic Materials, vol. 409, pp. 10-19, 2016.

[7] F. Ali, N. A. Sheikh, I. Khan, and M. Saqib, "Magnetic field effect on blood flow of Casson fluid in axisymmetric cylindrical tube: a fractional model," Journal of Magnetism and Magnetic Materials, vol. 423, pp. 327-336, 2017.

[8] D. Vieru, C. Fetecau, and C. Fetecau, "Flow of a viscoelastic fluid with the fractional Maxwell model between two side walls perpendicular to a plate," Applied Mathematics and Computation, vol. 200, no. 1, pp. 459-464, 2008.

[9] A. Shakeel, S. Ahmad, H. Khan, and D. Vieru, "Solutions with Wright functions for time fractional convection flow near a heated vertical plate," Advances in Difference Equations, vol. 2016, no. 1, p. 51, 2016.

[10] F. Ali, N. A. Sheikh, I. Khan, and M. Saqib, "Solutions with Wright function for time fractional free convection flow of Casson fluid," Arabian Journal for Science and Engineering, vol. 42, no. 6, pp. 2565-2572, 2017.

[11] M. Caputo and M. Fabrizio, "A new definition of fractional derivative without singular kernel," Progress in Fractional Differentiation and Applications, vol. 1, no. 2, pp. 1-13, 2015.

[12] J. Hristov, "Derivatives with non-singular kernels from the Caputo-Fabrizio definition and beyond: appraising analysis with emphasis on diffusion models," Frontiers in Fractional Calculus, vol. 1, pp. 270-342, 2017. 
[13] F. Ali, M. Gohar, I. Khan, N. A. Sheikh, S. A. A. Jan, and M. Saqib, "Magnetite molybdenum disulphide nanofluid of grade two: a generalized model with caputo-fabrizio derivative," in Microfluidics and Nanofluidics, intechopenhttps:// www.intechopen.com/, 2018.

[14] C. Fetecau, A. A. Zafar, D. Vieru, and J. Awrejcewicz, "Hydromagnetic flow over a moving plate of second grade fluids with time fractional derivatives having non-singular kernel," Chaos, Solitons \& Fractals, vol. 130, p. 109454, 2020.

[15] K. A. Abro, A. D. Chandio, I. A. Abro, and I. Khan, "Dual thermal analysis of magnetohydrodynamic flow of nanofluids via modern approaches of caputo-fabrizio and atanganabaleanu fractional derivatives embedded in porous medium," Journal of Thermal Analysis and Calorimetry, vol. 135, no. 4, pp. 2197-2207, 2018.

[16] N. A. Sheikh, F. Ali, I. Khan, and M. Saqib, "A modern approach of Caputo-Fabrizio time-fractional derivative to MHD free convection flow of generalized second-grade fluid in a porous medium," Neural Computing and Applications, vol. 30, no. 6, pp. 1865-1875, 2018.

[17] A. Atangana and D. Baleanu, "New fractional derivatives with nonlocal and non-singular kernel: theory and application to heat transfer model," Thermal Science, vol. 20, no. 2, pp. 763-769, 2016.

[18] A. Atangana and I. Koca, "Chaos in a simple nonlinear system with Atangana-Baleanu derivatives with fractional order," Chaos, Solitons \& Fractals, vol. 89, pp. 447-454, 2016.

[19] N. A. Sheikh, F. Ali, M. Saqib, I. Khan, and S. A. A. Jan, "A comparative study of Atangana-Baleanu and Caputo-Fabrizio fractional derivatives to the convective flow of a generalized Casson fluid," The European Physical Journal Plus, vol. 132, no. 1, p. 54, 2017.

[20] N. A. Sheikh, F. Ali, M. Saqib et al., "Comparison and analysis of the Atangana-Baleanu and Caputo-Fabrizio fractional derivatives for generalized Casson fluid model with heat generation and chemical reaction," Results in Physics, vol. 7, pp. 789-800, 2017.

[21] R. Chhabra and J. Richardson, Non-Newtonian Flow and Applied Rheology: Engineering Applications, Elsevier, Amsterdam, Netherlands, 2011.

[22] F. Ali, N. A. Sheikh, M. Saqib, and I. Khan, "Unsteady MHD flow of second-grade fluid over an oscillating vertical plate with isothermal temperature in a porous medium with heat and mass transfer by using the Laplace transform technique," Journal of Porous Media, vol. 20, no. 8, pp. 671-690, 2017.

[23] T. Sochi, "Flow of non-Newtonian fluids in porous media," Journal of Polymer Science Part B: Polymer Physics, vol. 48, no. 23, pp. 2437-2767, 2010.

[24] Y.-S. Wu and K. Pruess, "Chapter 2 Flow of non-Newtonian fluids in porous media," in Advances in Porous Media, M. Y. Corapcioglu, Ed., pp. 87-184, Elsevier, Amsterdam, Netherlands, 1996.

[25] S. De, J. A. M. Kuipers, E. A. J. F. Peters, and J. T. Padding, "Viscoelastic flow simulations in random porous media," Journal of Non-newtonian Fluid Mechanics, vol. 248, pp. 5061, 2017.

[26] F. Ali, N. A. Sheikh, M. Saqib, and A. Khan, "Hidden phenomena of an MHD unsteady flow in porous medium with heat transfer," Nonlinear Science Letters A, vol. 8, no. 1, pp. 101-116, 2017.

[27] B. Raftari, S. T. Mohyud-Din, and A. Yildirim, "Solution to the MHD flow over a non-linear stretching sheet by homotopy perturbation method," Science China Physics, Mechanics and Astronomy, vol. 54, no. 2, pp. 342-345, 2011.
[28] R. L. Bagley and P. J. Torvik, "A theoretical basis for the application of fractional calculus to viscoelasticity," Journal of Rheology, vol. 27, no. 3, pp. 201-210, 1983.

[29] T. Hayat, C. Fetecau, and M. Sajid, "On MHD transient flow of a Maxwell fluid in a porous medium and rotating frame," Physics Letters A, vol. 372, no. 10, pp. 1639-1644, 2008.

[30] Y.-S. Wu and K. Pruess, "A numerical method for simulating non-Newtonian fluid flow and displacement in porous media," Advances in Water Resources, vol. 21, no. 5, pp. 351-362, 1998.

[31] H. Darcy, Les fontaines publiques de la ville de Dijon (The public fountains of the city of Dijon), Dalmont, Paris, France, 1856.

[32] H. C. Brinkman, "The viscosity of concentrated suspensions and solutions," The Journal of Chemical Physics, vol. 20, no. 4, p. $571,1952$.

[33] S. V. Varma and M. S. Babu, "A Brinkman model for MHD viscous incompressible flow through a porous channel," Indian Journal of Pure and Applied Mathematics, vol. 16, no. 7, pp. 796-806, 1985.

[34] C. Lin and L. E. Payne, "Structural stability for a Brinkman fluid," Mathematical Methods in the Applied Sciences, vol. 30, no. 5, pp. 567-578, 2007.

[35] M. Asif, S. U. Haq, S. Islam, I. Khan, and I. Tlili, "Exact solution of non-Newtonian fluid motion between side walls," Results in Physics, vol. 11, pp. 534-539, 2018.

[36] A. Kumar, R. Singh, G. S. Seth, and R. Tripathi, "Double diffusive magnetohydrodynamic natural convection flow of brinkman type nanofluid with diffusion-thermo and chemical reaction effects," Journal of Nanofluids, vol. 7, no. 2, pp. 338-349, 2018.

[37] F. Ali, Aamina, I. Khan, N. A. Sheikh, M. Gohar, and I. Tlili, "Effects of different shaped nanoparticles on the performance of engine-oil and kerosene-oil: a generalized brinkman-type fluid model with non-singular kernel," Scientific Reports, vol. 8 , no. 1, p. 15285, 2018.

[38] S. A. A. Jan, F. Ali, N. A. Sheikh, I. Khan, M. Saqib, and M. Gohar, "Engine oil based generalized brinkman-type nano-liquid with molybdenum disulphide nanoparticles of spherical shape: Atangana-Baleanu fractional model," $\mathrm{Nu}$ merical Methods for Partial Differential Equations, vol. 34, no. 5, pp. 1472-1488, 2018.

[39] F. Aamina, I. Khan, and N. Sheikh Muhammad Saqib, "Magnetohydrodynamic flow of brinkman-type engine oil based MoS2-nanofluid in a rotating disk with hall effect," International Journal of Heat and Technology, vol. 35, no. 4, pp. 893-902, 2017.

[40] F. Ali, M. Gohar, and I. Khan, "MHD flow of water-based Brinkman type nanofluid over a vertical plate embedded in a porous medium with variable surface velocity, temperature and concentration," Journal of Molecular Liquids, vol. 223, pp. 412-419, 2016.

[41] F. Ali, S. A. A. Jan, I. Khan, M. Gohar, and N. A. Sheikh, "Solutions with special functions for time fractional free convection flow of Brinkman-type fluid," The European Physical Journal Plus, vol. 131, no. 9, p. 310, 2016.

[42] F. Ali, M. Gohar, I. Khan, N. A. Sheikh, and S. A. A. Jan, "Thermal radiation and magnetic field effects on different channel flows of CNTs brinkman-type nanofluids with water, kerosene and engine-oil," City University International Journal Of Computational Analysis, vol. 2, no. 1, 2018.

[43] F. Ali, I. Khan, and S. Shafie, "A note on new exact solutions for some unsteady flows of Brinkman-type fluids over a plane 
wall," Zeitschrift für Naturforschung A, vol. 67, no. 6-7, pp. 377-380, 2012.

[44] M. Caputo and J. M. Carcione, "A memory model of sedimentation in water reservoirs," Journal of Hydrology, vol. 476, pp. 426-432, 2013.

[45] F. Ali, M. Saqib, I. Khan, and N. Ahmad Sheikh, "Heat transfer analysis in ethylene glycol based molybdenum disulfide generalized nanofluid via atangana-baleanu fractional derivative approach," in Fractional Derivatives with MittagLeffler Kernel (Studies in Systems, Decision and Control), pp. 217-233, Springer, Berlin, Germany, 2019.

[46] M. Saqib, F. Ali, I. Khan, N. A. Sheikh, and A. Khan, "Entropy generation in different types of fractionalized nanofluids," Arabian Journal for Science and Engineering, vol. 44, no. 1, pp. 531-540, 2018.

[47] N. A. Sheikh, D. L. C. Ching, S. Ullah, and I. Khan, "Mathematical and statistical analysis of RL and RC fractional-order circuits," Circuits, Systems, and Signal Processing, vol. 31, pp. 1901-1915, 2012. 